\title{
A Typical Presentation of Tuberculous Pericarditis: Case Report
}

\author{
Faris Alhejaili MD FRCPC \\ Department of Medicine, Faculty of Medicine, King Abdulaziz University, Jeddah, Saudi Arabia
}

\begin{abstract}
A 23-year-old woman was admitted to the hospital for the management of progressive shortness of breath, fever and pleural effusion. Initial work up was negative for bacterial infection, tuberculosis and HIV. Further work up with echocardiographic study during admission revealed a moderate pericardial effusion ( $4 \mathrm{~cm}$ in thickness) but with no tamponade effect. Pericardiocentesis was performed and $1.2 \mathrm{~L}$ of sero-sanginousfluid was drained. All tumour and autoimmune markers were unremarkable. Rapid acid-fast bacilli culture demonstrated the growth of Mycobacterium tuberculosis. She was started on anti-TB medications and tolerated them well. Follow-up echocardiographic study and chest $X$-ray showed no re-accumulation of pericardial or pleural fluid. Tuberculous pericarditis is rare in developed countries but may still be seen in immigrant population. The variable clinical presentation make early diagnosis challenging and require high index of suspension.
\end{abstract}

Keywords: Tuberculous pericarditis-pleural effusion-tuberculosis-pericardial effusion

\section{Introduction}

Tuberculosis (TB) is a potentially serious infectious disease. It spreads through an airborne route causing an epidemic worldwide. The global report in 2012 showed a development of 8.6 million new cases and 1.3 million deaths related to TB. However, a fall in the diagnosis of new cases of TB cases was observed worldwide. Despite the challenges of emerging drug resistance, the mortality from TB is dropping (1). The World Health Organization (WHO) reported in 2012 reported an annual TB incidence rate of 18/100,000 population in Saudi Arabia. There is no available data on the prevalence of tuberculous pericarditis in Saudi Arabia. Cardiac involvement as a manifestation of extra pulmonary TB is estimated to be approximately $0.5 \%$ (2). In developed countries only $4 \%$ of acute pericarditis case are attributed to TB (3). This case aims to highlight the importance of recognizing and diagnosing tuberculous pericarditis in a TB endemic region such as Saudi Arabia and the importance of being attentive of atypical clinical presentations.

\section{Case Report}

A 23 year old female nursing student presented to our emergency department with a progressive shortness of breath for 3 months, fever with night sweating for 3 months, cough productive of yellow sputum and $5 \mathrm{~kg}$ weight loss. She has no history of chest pain, no hemoptysis and symptoms oh heart failure. She has no previous history or risk factors for tuberculosis or Human Immunodeficiency Syndrome (HIV). The initial clinical examination showed a temperature of $39.3 \mathrm{C}$, heart rate of 129 beat per minute, blood pressure 131/79 and oxygen saturation of 95\% in room air. She has normal Jugular Venous Pressure (JVP), no palpable lymph nodes, and normal cardiovascular examination. Her chest examination revealed tracheal shift to the right, decrease chest expansion, dullness on percussion on the right side and decreased breath sounds bilaterally. Initial blood work is reviewed in (table 1). Chest X-ray (CXR) on presentation showed massive left-sided pleural effusion, and mediastinal shift to the right (figure 1). After admission, diagnostic and therapeutic thoracentesis was performed. Pleural fluid analysis showed an exudative fluid with white blood cells count of 2550 cell $/ \mathrm{ml}$ consisting of $65 \%$ monocytes and $35 \%$ lymphocytes. Cytology was negative for malignancy. Fluid cultures were negative. Work up for TB was negative, with negative skin test, negative plural fluid acid-fast bacilli smear and fluid PCR. Pleural biopsy also came back inconclusive. The patient was started on empirical antibiotics with no improvement. Computed tomography (CT) of the chest arranged and showed re accumulation of the massive pleural effusion, extensive mediastinal lymphadenopathy and a moderate pericardial effusion (figure2). After the CT scan, a surgical chest tube was inserted and bronchoscopy was done. Bronchioalveolar (BAL) fluids were negative for AFB and PCR for TB in addition to negative bacterial and viral cultures. BAL fluid cytology was also negative. Further evaluation of the pericardial effusion was done with an echocardiography that thickening posterior pericardium, moderate pleural effusion and preserved left ventricular functions. The patient was still febrile and not responding to antibiotics. Subsequently she started to develop orthopnea and lower limb edema. Her CXR showed bilateral pleural effusion (figure 3). After increasing her diuretics doses, the decision was made to perform a pericardiocentesis for diagnostic and therapeutic purposes. 1.2 liter of sero-sanginous fluid drained followed by a moderate clinical improvement but she continued to be febrile. Pericardial fluid came back positive for rapid AFB and later TB PCR came back positive as well. Anti-TB medication (rifampicin, isoniazid, pyrazinamide, ethambutol), pyridoxine, and a 4-week course of steroids were commenced. Clinical improvement was noticeable within 72 hours. A weak later, her SOB resolved completely and she was discharged home with clinic follow up. CXR 2 weeks after discharge was clear with no recurrence of her pleural effusion.

\section{Discussion}

The clinical presentation of TB pericarditis is variable and non-specific.Symptoms include fever, weight loss, fatigue and night sweats. More common clinical symptoms are cough, SOB and chest pain(4). Patients can present acutely 


\section{International Journal of Science and Research (IJSR) \\ ISSN (Online): 2319-7064 \\ Index Copernicus Value (2013): 6.14 | Impact Factor (2015): 6.391}

with cardiac tamponade(5), but may also present with heart failure from chronic cardiac compression(4). The variable clinical presentation makes the condition difficult to diagnose initially.

TB pericarditis have four stages: (1) dry stage with exudation of fibrinous material and early immune response; (2) effusive stage of sero-sanguineous fluid; (3) absorptive stage with organization of granulomatous caseation and pericardial thicking; (4) constrictive stage caused by scarring. Our case was likely in the effusive stage. The fluid drained was seros-anguineous which is typical in TB pericarditis(4, 5). Our initial suspicion was bacterial infection due to the nature of the presentation and the absence of risk factors for HIV, TB and malignancy. Literature reviewrevealed a few cases reports documenting the presence of massive amount of pericardial effusion or 4 cm pericardial thickness(6-8).

AFB smear, cultures and PCR have variable detection rates. Direct Ziehl-Neelsen stain has a poor detection rate of $0 \%$ to $42 \%$ only (4). Cultures of pericardial effusion positive results are seen in only 55 - 93\% of patients with TB pericarditis $(3,9)$. Cegielski et al. compared PCR, culture and histopathology in diagnosing TB pericarditis (9). TB was correctly diagnosed by culture in $93 \%$ of patients as compared with $81 \%$ by PCR and $87 \%$ by histology. They found that with the use of PCR, the sensitivity was higher for tissue specimens as compared with fluid specimens (9).

For the diagnosis of tuberculous pericarditis, pericardial fluid should be obtained for analysis. Typical TB effusions are exudative. Adenosine deaminase (ADA) activity andinterferon (IFN)- $\gamma$ levels are useful diagnostic tools. Elevation of ADA activity $\geq 40 \mathrm{U} / \mathrm{l}$ had $87 \%$ sensitivity and $89 \%$ specificity. IFN- $\gamma \geq 50 \mathrm{pg} / \mathrm{ml}$ concentration had $92 \%$ sensitivity, $100 \%$ specificity and a positive predictive value (PPV) of $100 \%$ (10).

The recommended treatment coarse for TB pericarditis is at least 6 months (11). The role of concomitant steroid use is controversial. A review done by Ntsekhe et al. found that the use of steroids in addition to the standard anti-TB medications reduces the death rate and the reaccumulation of pericardial effusion (12).

\section{Conclusion}

Tuberculous pericarditis is an uncommon manifestation of TB. Its incidence reduced dramatically in the developed countries, but it may still present a diagnostic challenge in immigrant population and endemic areas. The variable clinical presentation makes the diagnosis challenging at the initial presentation, but a meticulous history and a general approach to such an unusual presentation is crucial in diagnosing and treating such disease.
Table 1: Summery $\mathrm{f}$ initial blood work

\begin{tabular}{|c|c|c|}
\hline Variable & Value & Normal range \\
\hline White blood cells count & 4.8 & $4.8-10.8 \times 10^{9} / \mathrm{L}$ \\
\hline Hemoglobin & 12.7 & $12-16 \mathrm{~g} / \mathrm{dL}$ \\
\hline Platelets & 357 & $130-400 \times 10^{9} \mathrm{~L}$ \\
\hline Blood Urea Nitrogen & 1.6 & $2.5-8 \mathrm{mmol} / \mathrm{L}$ \\
\hline Creatinin & 65 & $50-90 \mathrm{mmol} / \mathrm{L}$ \\
\hline Albumin & 35 & $35-50 \mathrm{~g} / \mathrm{L}$ \\
\hline Total protein & 86 & $60-80 \mathrm{~g} / \mathrm{L}$ \\
\hline ESR & 19 & $<10 \mathrm{~mm} / \mathrm{h}$ \\
\hline HIV & Negative & $\mathrm{NA}$ \\
\hline
\end{tabular}

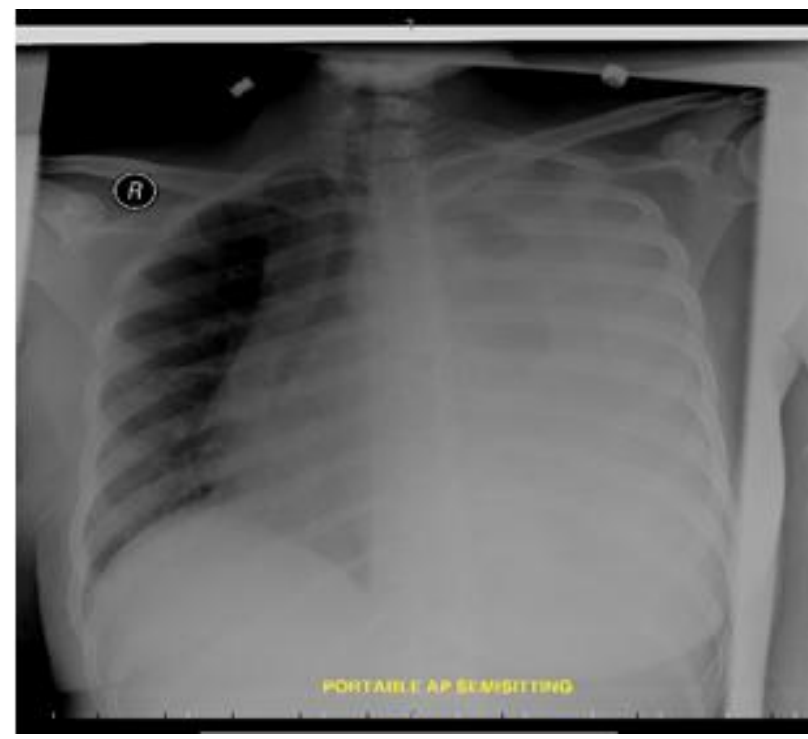

Figure 1: Initial CXR

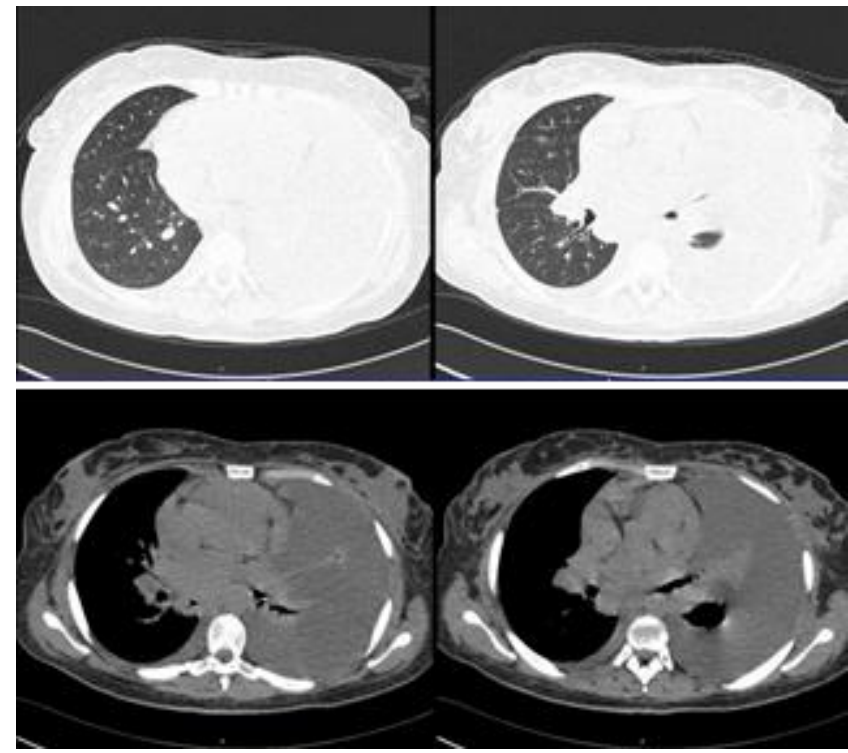

Figure 2: Selected images of CT Chest in lung and mediastinal window 


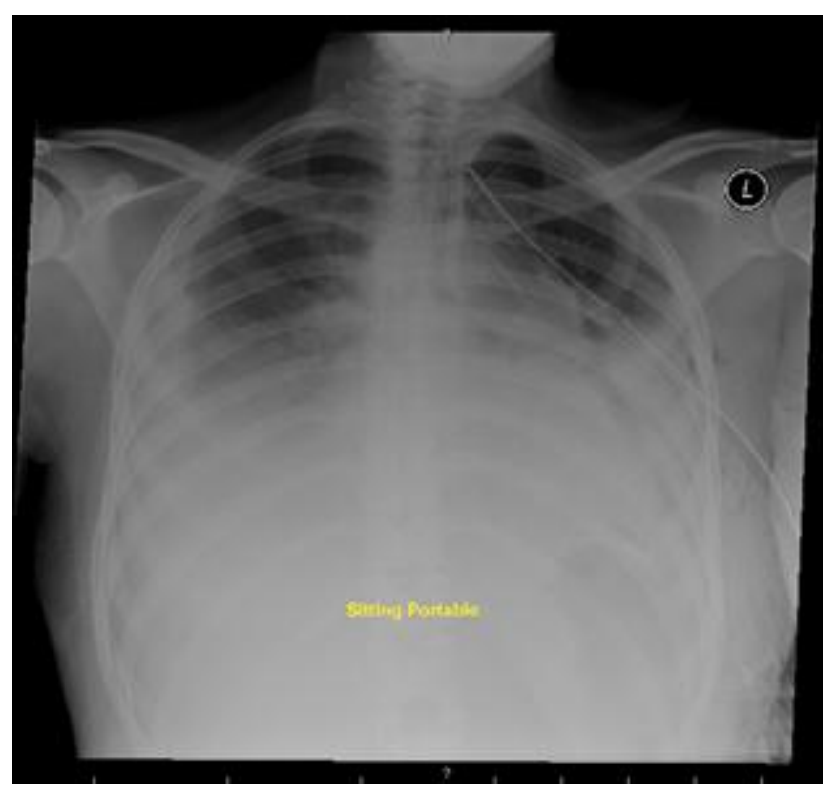

Figure 3: CXR after surgical chest tube insertion

\section{References}

[1] Zumla A, George A, Sharma V, Herbert N, Baroness Masham of Ilton. WHO's 2013 global report on tuberculosis: successes, threats, and opportunities. Lancet. 2013;382(9907):1765-7.

[2] Roubille F, Cayla G, Gahide G. Cardiac tumor mimicking acute myocardial infarction. Arch Cardiovasc Dis. 2008;101(10):677-8.

[3] Sagristà-Sauleda J, Permanyer-Miralda G, Soler-Soler J. Tuberculous pericarditis: ten year experience with a prospective protocol for diagnosis and treatment. J Am Coll Cardiol. 1988;11(4):724-8.

[4] Mayosi BM, Burgess LJ, Doubell AF. Tuberculous pericarditis. Circulation. 2005;112(23):3608-16.

[5] Sharma SK, Mohan A. Extrapulmonary tuberculosis. Indian J Med Res. 2004;120(4):316-53.

[6] Avşar A, Günay NK, Celik A, Melek M. A case of cardiac tamponade caused by tuberculous pericarditis. Turk Kardiyol Dern Ars. 2008;36(7):482-4.

[7] Heller T, Lessells RJ, Wallrauch C, Brunetti E. Tuberculosis pericarditis with cardiac tamponade: management in the resource-limited setting. Am J Trop Med Hyg. 2010;83(6):1311-4.

[8] Massoure PL, Boddaert G, Caumes JL, Gaillard PE, Lions C, Grassin F. Porridge-like tuberculous cardiac tamponade: treatment difficulties in the Horn of Africa. Gen Thorac Cardiovasc Surg. 2010;58(6):276-8.

[9] Cegielski JP, Devlin BH, Morris AJ, Kitinya JN, Pulipaka UP, Lema LE, et al. Comparison of PCR, culture, and histopathology for diagnosis of tuberculous pericarditis. J Clin Microbiol. 1997;35(12):3254-7.

[10] Reuter H, Burgess L, van Vuuren W, Doubell A. Diagnosing tuberculous pericarditis. QJM. 2006;99(12):827-39.

[11]Lee VY, Wong JT, Fan HC, Yeung VT. Tuberculous pericarditis presenting as massive haemorrhagic pericardial effusion. BMJ Case Rep. 2012;2012.

[12] Ntsekhe M, Wiysonge C, Volmink JA, Commerford PJ, Mayosi BM. Adjuvant corticosteroids for tuberculous pericarditis: promising, but not proven. QJM. 2003;96(8):593-9. 